\title{
A novel mutation in NCF2 resulting in very-early-onset colitis and juvenile idiopathic arthritis in a patient with chronic granulomatous disease
}

Suzan AlKhater ${ }^{1,2^{*}}$ (B)

\begin{abstract}
Background: Chronic granulomatous disease (CGD) is a rare primary immunodeficiency disorder caused by a defect in the nicotinamide adenine dinucleotide phosphate (NADPH) oxidase complex. The disease primarily presents with recurrent infections, and patients may also present with inflammatory conditions, including noninfectious colitis, and an increased frequency of autoimmunity. We report here a patient with CGD in whom the presentation, unlike the classical presentation of CGD, was predominantly of an inflammatory and autoimmune phenotype.

Case presentation: A 3-year-old Pakistani female presented with bloody diarrhea since the age of 7 days, followed by the development of perianal abscesses and fistula. There was no other history of recurrent infections. The patient subsequently developed joint pain and stiffness with persistently elevated inflammatory markers and elevated anticyclic citrullinate peptide (anti-CCP) antibody titer. She was diagnosed with oligoarticular juvenile idiopathic arthritis and colitis. The diagnosis of CGD was later made and was based on the absence of NADPH oxidase activity in the patient's neutrophils upon phorbol myristate acetate (PMA) stimulation using the dihydrorhodamine-1,2,3 (DHR) flow cytometry test. Targeted next-generation sequencing revealed an unreported deletion mutation in exon 10 as a homozygous loss-of-function variant of the human neutrophil oxidase factor 2 (NCF2) (NCF2: NM_001190789, nucleotide change: c.855_856del:p.T285fs). The gene encodes a protein subunit, p67 phox , in the NADPH enzyme complex.
\end{abstract}

Conclusions: The case emphasizes the importance of maintaining high clinical suspicion of immunodeficiency and CGD in patients with very-early-onset colitis and autoimmune disorders. This case is important due to its rarity and because it might represent a previously undiscovered mutation, which is possibly more common in the patient's ethnic group. Other mutations in NCF2 have been linked to inflammatory bowel disease and autoimmunity, but without CGD, suggesting similarities in the pathogenesis.

Keywords: Chronic granulomatous disease, Primary immunodeficiency, Early-onset colitis, Inflammatory bowel disease, Juvenile idiopathic arthritis

\footnotetext{
*Correspondence: Saalkhater@iau.edu.sa

${ }^{1}$ Department of Pediatrics, College of Medicine, Imam Abdulrahman Bin

Faisal University, Dammam, Saudi Arabia

Full list of author information is available at the end of the article
} 


\section{Background}

Primary immunodeficiency disorders (PIDs) are an inherited group of disorders characterized by a unique combination of the inability to fight pathogens, resulting in recurrent infections, and immune dysregulation causing autoimmune diseases $[1,2]$.

Chronic granulomatous disease (CGD) is a rare PID with an incidence of 1:250,000 live births in Western countries [2]. The disease is usually severe and diagnosed in infancy, with the only curative treatment being hematopoietic stem cell transplantation, although gene therapy and gene editing are promising alternatives $[3,4]$.

CGD is caused by a defect in phagocytic neutrophils that inhibits the capability of eliminating intracellular pathogens [2]. This is caused by mutations that affect the nicotinamide adenine dinucleotide phosphate (NADPH) oxidase complex, a transmembrane protein that clears pathogens by generating reactive oxygen species (ROS) [2]. X-linked mutations (XL-CGD), are limited mostly to males and are located on the $C Y B B$ gene that codes for gp91-phox protein [4]. The XL form of CGD account for approximately two-thirds of cases of in Westerners [5]. The mutations that cause autosomal recessive CGD (AR-CGD) encode the components of the phagocytic $\mathrm{NADPH}$ oxidase complex $-\mathrm{p} 22^{\text {phox }}, \mathrm{p} 47^{\text {phox }}, \mathrm{p} 67^{\text {phox }}$ $\mathrm{p} 40^{\text {phox }}$, and EROS subunits-and are located in genes $C Y B A, N C F 1, N C F 2, N C F 4$ and $C Y B C 1$, respectively [5, 6]. The AR forms of CGD are more commonly reported in studies from Turkey [7], Israel [8], Arab countries and North Africa [9], Iran [10], and India [11], presumably because of the higher rate of consanguineous marriage (Table 1).

Of all the mutations associated with CGD, mutations in the NCF2 gene that affect $\mathrm{p} 67^{\text {phox }}$ expression (p67phox) are the rarest and are previously reported as clinically milder than others [9]. In Western populations, approximately $6 \%$ of CGD cases are caused by defects in p67 ${ }^{\text {phox }}$ [5], a 526-amino acid protein encoded by NCF2 (gene locus 1q25) [https://www.omim.org/entry/608515].

In addition to susceptibility to recurrent bacterial and fungal infections, inflammatory manifestations are common in CGD. An increased overall risk of developing malignancies has previously been reported in CGD patients [12]. However, this was not supported by findings from recent large cohorts studies $[7,9]$. The sporadic cases reported may not present a true causeeffect relationship $[9,13]$. On the other hand, among the inflammatory manifestations of CGD, intestinal involvement in the form of diffuse hyperinflammation is the commonest, in both XL and AR forms, and may reach a prevalence of $50 \%$ [14]. Patients with CGD appear to be at an elevated risk of developing colitis that is similar to Crohn's type of inflammatory bowel disease (IBD). In fact, severe intestinal inflammation may be the first clinical sign of CGD, as observed in patients with very-early-onset disease (defined as onset at younger than 6 years of age) [15]. CGD colitis is histologically indistinguishable from classical Crohn's disease and presents with similar signs, including growth failure, anemia and failure to thrive, abdominal pain, diarrhea,

Table 1 Summary of $\mathrm{X}$-linked and autosomal recessive forms of chronic granulomatous disease genes according to ethnic origin

\begin{tabular}{|c|c|c|c|c|}
\hline Publication [reference] & Number and population & X-linked ${ }^{a}$ & Autosomal recessive $^{\mathbf{b}}$ & $\begin{array}{l}\text { Type } \\
\text { of autosomal } \\
\text { recessive gene }\end{array}$ \\
\hline Köker et al. [7] & 89 Turkish & $34(38.2 \%)$ & $50(56.2)$ & $\begin{array}{l}\text { CYBA }(22.5 \%) \\
\text { NCF1 }(19.1 \%) \\
\text { NCF2 }(14.6 \%)\end{array}$ \\
\hline \multirow[t]{5}{*}{ Van den Berg et al. [9] } & 357 European & $265(74.2 \%)$ & $92(25.7 \%)$ & \multirow{5}{*}{$\begin{array}{l}\text { NCF1 }(16 \%) \\
\text { CYBA }(5.1 \%) \\
\text { NCF2 }(2.5 \%) \\
\text { Unknown }(8.6 \%\end{array}$} \\
\hline & 12 Turkey $^{c}$ & $4(33 \%)$ & $8(67 \%)$ & \\
\hline & 38 Arab/North Africanc & $11(29 \%)$ & $27(71 \%)$ & \\
\hline & 6 East and South Asia & $1(17 \%)$ & $5(83 \%)$ & \\
\hline & 16 Israeli/Jew & $9(56 \%)$ & $7(44 \%)$ & \\
\hline Wolach et al. [8] & 84 Israeli (Jew, Arabc , visitor) & $32(38 \%)$ & $\begin{array}{l}52(62 \%)^{c} \\
(64 \% \text { consanguinity) }\end{array}$ & $\begin{array}{l}\text { NCF1 (31\%) } \\
\text { NCF2 (19\%) } \\
\text { CYBA (12\%) }\end{array}$ \\
\hline Fattahi et al. [10] & 93 Iranianc & $12(12.9 \%)$ & $81(87.1 \%)$ & NCF1 (55.5\%) \\
\hline Kulkarni et al. [11] & $\begin{array}{l}90 \text { Indian }^{c} \\
\text { (32\% consanguinity) }\end{array}$ & $27(30 \%)$ & $63(70 \%)$ & $\begin{array}{l}\text { NCF1 }(56 \%) \\
\text { CYBA (7\%) } \\
\text { NCF1 }(7 \%)\end{array}$ \\
\hline
\end{tabular}

\footnotetext{
${ }^{a}$ CYBB $,{ }^{b} N C F 1, C Y B A, N C F 2, N C F 4$

c Reported high rate of consanguinity
} 
nausea, vomiting and constipation [15]. In addition, NCF2 mutations that allow for some production of ROS have been associated with susceptibility to autoimmune diseases, including systemic lupus erythematosus (SLE) $[16,17]$ and sarcoidosis [18].

We present a case in which a patient with early-onset colitis was later diagnosed with CGD and developed symptoms of arthritis. The constellation of disease in our patient is suggestive of another novel mutation in one of the genes that encodes the NADPH oxidase complex that predisposes patients to severe inflammation and autoimmunity.

\section{Case presentation}

Our patient is a 3-year-old Pakistani female born to firstdegree cousins. She presented at the age of 10 months with enlarged cervical lymph nodes and diarrhea. She had multiple admissions for recurrent diarrheal illnesses. The diarrhea started at the age of 7 days, with a frequency of more than 10 times per day of watery consistency but no blood or mucus. She was started on an elemental amino acid-based formula because of a diagnosis of food protein-induced enterocolitis syndrome based on a presentation of severe bloody diarrhea, acidosis and hypotension. She had a sister who had a similar presentation of chronic diarrhea and fever and died at the age of 1 year due to septic shock. Immune deficiency was not suspected and the underlying cause was not investigated. Also, no genetic tests were obtained and the family did not receive any prenatal genetic counseling.

After removing cow's milk from our patient's diet and initiating the special formula, her diarrhea settled, but at the age of 1 year, she had a recurrence of diarrhea described as bloody and associated with multiple perianal abscesses. The abscesses were treated with intravenous antibiotics and surgically drained. The culture from the drained pus repeatedly grew extended spectrum $\beta$-lactamase-producing E. coli and Klebsiella species. At the age of 2 years, she developed arthritis involving large joints of the lower limb (knees and ankle) and wrist associated with morning stiffness and an inability to walk.

On examination, the patient had severe failure to thrive. Multiple enlarged cervical lymph nodes were noted. The scar of the Bacillus Calmette-Guérin (BCG) vaccine was normal, and no axillary lymph nodes were detected. The liver was palpable $3 \mathrm{~cm}$ below the costal margins. She had swelling of the left knee joints with a reduced range of movement. Perineal examination revealed inflammation, multiple scars of drained perianal abscesses and a perianal fistula opening (Fig. 1).

Laboratory investigation revealed leukocytosis $\left(29.1 \times 10^{9} / \mathrm{L}\right.$ ), anemia (hemoglobin $8.4 \mathrm{~g} / \mathrm{dL}$ ), elevated

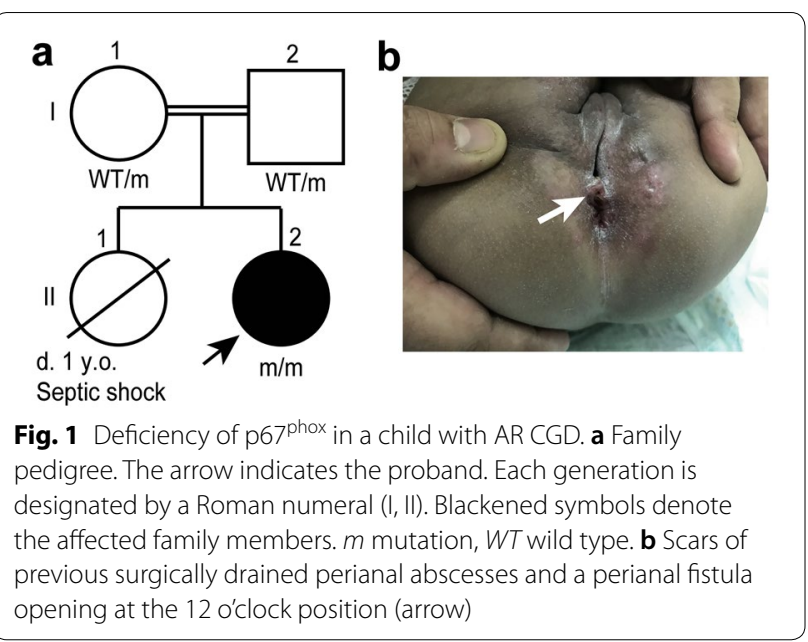

inflammatory markers (C-reactive protein $12.0 \mathrm{mg} / \mathrm{dL}$, and ESR $80 \mathrm{~mm} / \mathrm{h}$ ) and elevated immunoglobulin levels. A rheumatological workup was positive for serological markers of autoimmunity, including antinuclear antibodies (ANA) (titer 1:80, speckled pattern), rheumatoid factor $(25.1 \mathrm{IU} / \mathrm{mL}$; normal $<15)$, anti-cyclic citrullinate peptide (anti-CCP) antibody titer $(216.7 \mathrm{u} /$ $\mathrm{mL}$; normal $<20$ ) and anti-double-stranded DNA (anti-dsDNA) antibodies (83.6 IU $/ \mathrm{mL}$; normal $<35)$. In addition, these markers were positive on repeated testing. Other markers, including anti-RNP, anti-SSA, anti-SSB, and anti-Sm, remained negative, while C3 and C4 complement levels were not suppressed. Joint fluid sampling was obtained, and an infectious etiology for joint involvement was excluded by culture obtained from the knee joint. Based on the clinical presentation and laboratory workup, a diagnosis of oligoarticular juvenile idiopathic arthritis (JIA) was provided.

Furthermore, microbiological testing for infectious diarrhea, including stool culture and sensitivity tests, a stool ova and parasite examination, Clostridium difficile toxin test, and Giardia lamblia and Cryptosporidium stool antigen tests, were negative. The celiac panel was also negative, and malabsorption tests, including fecal fat and fecal elastase tests, were normal. Stool examination was positive for fecal occult blood, and fecal calprotectin, an indicator of intestinal inflammation, was elevated at $580 \mathrm{mcg} / \mathrm{g}$ (normal <50.0). Workup for TB was negative, including bone marrow and lymph node examination, using polymerase chain reaction (PCR) assay, cultures, and the TB gold test. In addition, a purified protein derivative (PPD) skin test was performed on the patient and showed no reaction.

In view of her presentation and the early colitis symptoms, she was further evaluated, and a diagnosis of CGD was made based on the oxidative burst assay [dihydrorhodamine-1,2,3 (DHR) flow cytometry test], 
which revealed an absence of NADPH oxidase activity in the patient's neutrophils upon phorbol myristate acetate (PMA) stimulation (Fig. 2). The neutrophil oxidative index (NOI) of the patient was $<5$. NOI measures the oxidative ability of phagocytes and their overall integrity and is calculated from the ratio of the fluorescence in stimulated phagocytic cells to the fluorescence expressed in unstimulated phagocytic cells [19]. The normal value in control specimens is usually $>73$ [19].

The diagnosis of CGD was confirmed by targeted next-generation sequencing using Ion AmpliSeq Designer software (Life Technologies, Carlsbad, CA, USA). The gene panel used analyzed over 200 genes associated with immune deficiency and immune dysregulation, including auto-Inflammatory and Autoimmunity syndromes. The test was performed on venous blood samples collected in EDTA tubes for DNA extraction after obtaining written consent from the parents to perform the genetic diagnostic assay. The test revealed a deletion mutation in exon 10 as a homozygous loss-of-function variant of NCF2 (NCF2: NM_001190789, nucleotide change: c.855_856del:p. T285fs). Both parents were heterozygous for the mutation. We believe this to be a novel variant that has not been previously reported.

To prevent serious and life-threatening infections, the patient was started on an antibacterial and antifungal prophylactic regimen consisting of trimethoprim-sulfamethoxazole (trimethoprim $6 \mathrm{mg} /$ $\mathrm{kg} /$ day) and itraconazole $(5 \mathrm{mg} / \mathrm{kg} /$ day), respectively. She remained free of infection. Her arthritis symptoms were initially managed with naproxen followed by oral prednisolone at a dose of $1 \mathrm{mg} / \mathrm{kg} /$ day for 4 weeks followed by gradual tapering and then discontinued after 4 months. In addition, hydroxychloroquine was initiated and continued throughout the observation period, which lasted for 18 months following the onset of arthritis. She showed improvement and started to walk with no pain and had no relapse of symptoms during subsequent follow-up visits. However, we failed to establish long-term follow-up. In addition, further endoscopic, histological and imaging studies were planned but not performed because the patient and family traveled to their home country and were lost to follow-up.
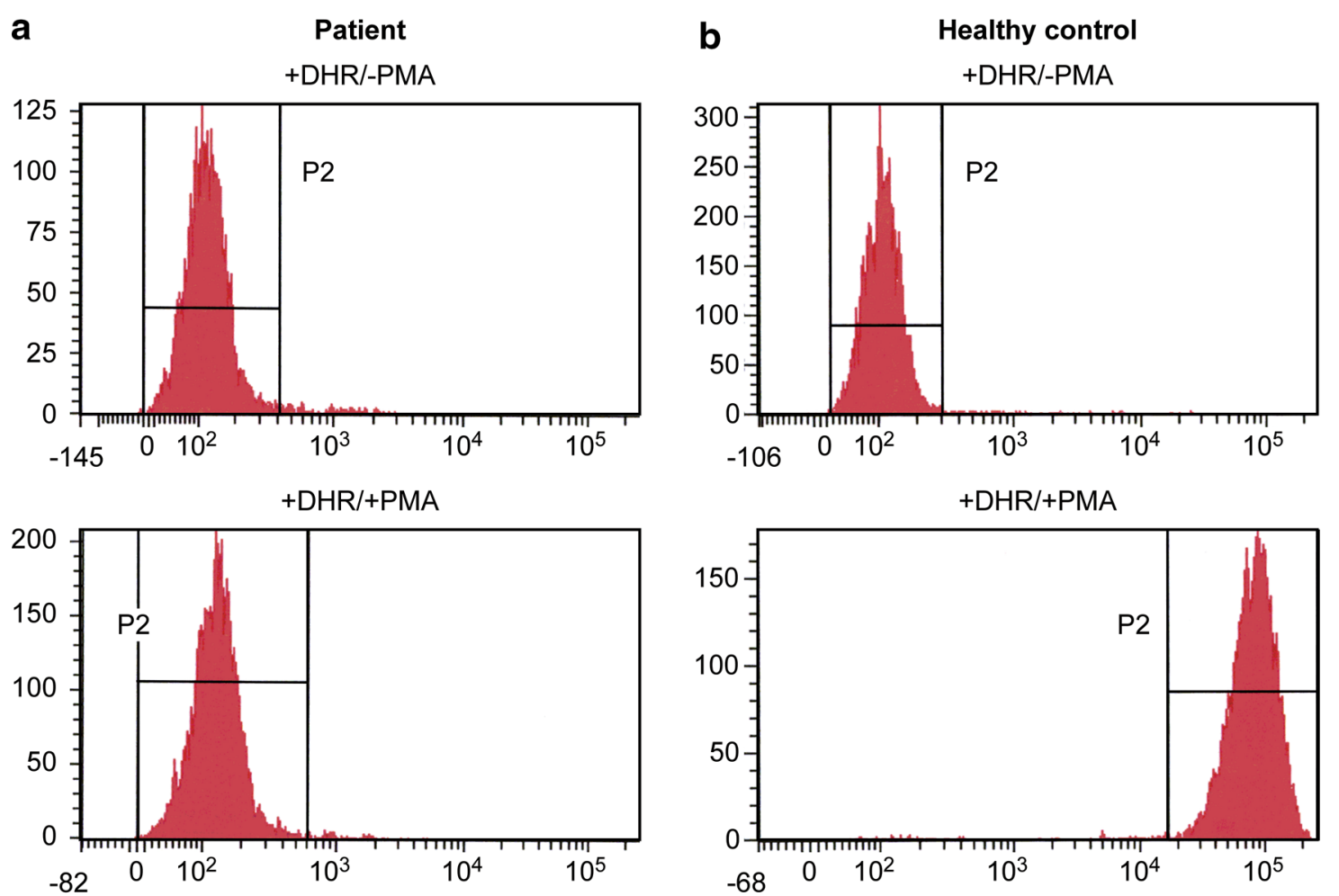

Fig. 2 Neutrophil oxidative burst test using DHR. The top graphs represent the unstimulated cells, while the bottom graphs are cells stimulated using PMA. a The patient showed an absence of reactivity after PMA stimulation, consistent with CGD. $\mathbf{b}$ Normal neutrophil respiratory burst showing a complete shift in fluorescence after stimulation in a healthy control. DHR dihydrorhodamine-1,2,3; PMA phorbol myristate acetate; CGD chronic granulomatous disease 


\section{Discussion and conclusions}

We report a case of CGD with a rare presentation of very-early-onset intestinal inflammation and an autoimmune disorder, JIA. The diagnosis of oligoarticular JIA was made according to the widely used International League of Associations for Rheumatology Classification [20], which defines JIA as the presence of arthritis in a child under 16 years of age and lasting at least 6 weeks. Oligoarticular JIA involves fewer than 5 joints during the first 6 months of disease. Our patient was positive for autoimmune antibodies, including ANA, commonly seen in oligoarticular JIA. In addition, tests for other autoantibodies were positive, including that for antidsDNA, despite the absence of manifestation of other connective tissue disorders, including lupus. JIA is associated with the formation of various autoantibodies and the development of other connective tissue diseases [21].

Notably, apart from perianal abscesses, which is likely a presentation of CGD colitis, history of other recurrent infections were unreported in our patient. Nevertheless, the pattern of organisms isolated in the patient points to a defective phagocytic component of the immune system, which results in a predisposition to gramnegative organisms, particularly E. coli and Klebsiella [1]. Furthermore, the early symptoms soon after birth in our patient were likely signs of CGD with intestinal inflammation. The presenting symptoms can be mistaken for food hypersensitivity reactions.

The case is important due to its rarity and because it might represent a previously undiscovered mutation. In addition, it is interesting that the mutation may be more common in the patient's ethnic group, as other mutations in the NCF2 gene have been reported in cases from the Middle East and South Asia but not in Western populations.

Similar to our case, Vignesh et al. [22] described three patients from India with a diagnosis of CGD due to NCF2 defects. All 3 patients had signs of colitis from early infancy, while in two of the three cases, colitis was an initial presenting feature of the disease [22]. One child, a 2-year-old boy, who was ill since birth and developed fulminant colitis at $1 \frac{1}{2}$ years, had a homozygous deletion in NCF2 (c835_836delAC:p. T279fsX294), which resulted in a stop codon in exon 10 and reduced expression of the p67 ${ }^{\text {phox }}$ protein. In addition, all the reported cases had failure to thrive, pneumonia and granulomas. Similar presentations have been reported in Iranian [23] and Turkish children [7] with CGD due to NCF2 defects.

CGD associated colitis was reported in patients with CGD since the disease was first described. However, specific mutations that are associated with the development of colitis have been reported only recently in CGD patients [7]. Köker et al. [7] noted that patients with the $\mathrm{p} 67^{\mathrm{phox}}$ phenotype had an earlier clinical presentation and were diagnosed at a younger age. Marciano et al. [15] noted GI involvement in 46 of 140 CGD patients (33\%), which was more common in XL-CGD. Magnani et al. [24] described the intestinal manifestations of CGD in a cohort of 98 patients; $70 \%$ of the cohort had inflammatory episodes, and half of the patients had AR-CGD.

The similarities between Crohn's disease and CGD patients presenting with colitis may lead to misdiagnosis. Interestingly, mutations affecting the NADPH oxidase complex that involve early-onset colitis, but without CGD, are reported in the literature. Single nucleotide polymorphisms (SNPs) associated with an increased risk of IBD are also located in the genes that encode the NADPH oxidase complex [25]. For many of these mutations, the effect on ROS formation may be insufficient to cause CGD, but the clinical and pathological features of colitis in CGD and Crohn's disease suggest similarities in pathogenesis [26-28].

Interestingly, a meta-analysis of genome-wide association studies revealed that many loci for susceptibility to IBD overlap with those of other inflammatory and immune-mediated diseases, including PIDs [29]. In fact, many monogenic disorders of the immune system can manifest with immune dysregulation, resulting in an aberrant immune system that is unable to fight pathogens, yet fights against selftissue, causing autoimmunity [2, 30]. Therefore, although viewed as paradoxical in the past, the combination of immunodeficiency and autoimmune disorders is now understood to be interrelated, with common mechanisms that might be considered "two sides of the same coin" [31].

Indeed, sporadic cases of CGD presenting with autoimmune disorders appeared in the literature. Chou et al. [16] described a child with SLE (a child of consanguineous parents of Arab origin) who had chronic diarrhea since the age of 3 months and later developed failure to thrive, chronic arthralgias, and stiffness in multiple joints. The symptoms of inflammation and autoimmunity delayed a diagnosis of CGD, which was revealed after genetic testing that showed mutations in the NCF2 gene. In addition, JIA was recently reported in an Iranian child with CGD [32].

Equally important to the IBD gene studies was the identification of specific mutations in NCF2 that have been considered risk factors for SLE, without an associated immune deficiency or CGD manifestation [33-36]. Although no similar genetic links were reported in rheumatoid arthritis (RA), a link was recently identified between NCF2 and RA 
by studying the interaction of the various subunits of the neutrophil's NADPH oxidase complex during its assembly process. Aberrant neutrophils were found to be the disease-causing agent in RA [37]. Defects in NADPH oxidases in neutrophils were found to result in the citrullination of specific proteins, including the citrullination of type II collagen, resulting in the autoantibody formation and autoimmunity observed in RA [37-39]. Moreover, the citrullination of p67 phox/ NCF2 result in the inability to further assemble the NADPH oxidase complex, therefore disrupting the synthesis of ROS [37]. ROS are known for their antiinflammatory properties and have been reviewed elsewhere [40]; their deficiency has been implicated in the pathogenesis of inflammatory autoimmune diseases.

Although these mechanisms may operate differently in CGD patients, the combination of colitis and RA, or JIA, in a CGD patient, as seen here, suggests that NCF2 has immunomodulating effects. As a result of failure to assemble the protein subunits of the NADPH complex in CGD and deficient ROS production, the immune system is unable to eradicate pathogens while being at high risk for autoimmunity.

In conclusion, our case illustrates the different facets of PIDs and demonstrates that their presentation is not limited to a predisposition to repeated infections. The predominance of inflammatory manifestations in our patient emphasizes the importance of correctly recognizing the different symptoms and various presentations of PIDs and not overlooking the possibility of complex involvement of intestinal inflammatory manifestations and autoimmunity in patients with CGD. The discovery of more cases of CGD associated with the NCF2 mutation will be necessary to further characterize this mutation and understand the complex phenotypic associations.

\begin{abstract}
Abbreviations
JIA: oligoarticular juvenile idiopathic arthritis; BCG: Bacillus Calmette-Guérin; PCR: polymerase chain reaction; PPD: purified protein derivative; DHR: dihydrorhodamine-1,2,3; PMA: phorbol myristate acetate; NOI: neutrophil oxidative index; NADPH: nicotinamide adenine dinucleotide phosphate; CRP: $C$ reactive protein; NCF2: human neutrophil oxidase factor 2 ; CGD: chronic granulomatous disease; AR-CGD: autosomal recessive; XL-CGD: $X$-linked; SNPS: single nucleotide polymorphisms; IBD: inflammatory bowel disease; PIDs: primary immunodeficiency disorders; SLE: systemic lupus erythematosus; ANA: antinuclear antibodies; anti-dsDNA: anti-double-stranded DNA; RA: rheumatoid arthritis; CCP: cyclic citrullinate peptide.
\end{abstract}

\section{Acknowledgements}

Not applicable.

\section{Authors' contributions}

SA designed the study, performed the data interpretation and the preparation of the manuscript. The author read and approved the final manuscript.
Funding

Not applicable.

\section{Availability of data and materials}

Any additional data about the materials and methods will be provided by the corresponding authors upon request.

\section{Ethics approval and consent to participate}

This study followed guidelines from the Declaration of Helsinki and was approved by the Ethics Committee at King Fahad University Hospital, Al-Khobar, Saudi Arabia. The patient and parents were included in the study after written informed consent was obtained from the parents and signed informed consent documentation to provide peripheral blood by a single needle puncture.

\section{Consent for publication}

Additional informed consent was obtained for publication from all individual participants for whom identifying information is included in this article.

\section{Competing interests}

The author declares no competing interests.

\section{Author details}

${ }^{1}$ Department of Pediatrics, College of Medicine, Imam Abdulrahman Bin Faisal University, Dammam, Saudi Arabia. ${ }^{2}$ King Fahad Hospital of the University, P.O, Box 2208, Al-Khobar 31592, Saudi Arabia.

Received: 25 August 2019 Accepted: 5 November 2019

Published online: 21 November 2019

\section{References}

1. Alkhater SA. Approach to the child with recurrent infections. J Fam Commun Med. 2009;16:77-82.

2. Amaya-Uribe L, Rojas M, Azizi G, Anaya JM, Gershwin ME. Primary immunodeficiency and autoimmunity: a comprehensive review. J Autoimmun. 2019;99:52-72.

3. Seger RA. Modern management of chronic granulomatous disease. $\mathrm{Br}\rfloor$ Haematol. 2008;140:255-66.

4. Arnold DE, Heimall JR. A review of chronic granulomatous disease. Adv Ther. 2017;34:2543-57.

5. Roos D, Kuhns DB, Maddalena A, Bustamante J, Kannengiesser C, de Boer $\mathrm{M}$, et al. Hematologically important mutations: the autosomal recessive forms of chronic granulomatous disease (second update). Blood Cells Mol Dis. 2010:44:291-9.

6. Thomas DC, Clare S, Sowerby JM, Pardo M, Juss JK, Goulding DA, et al. Eros is a novel transmembrane protein that controls the phagocyte respiratory burst and is essential for innate immunity. J Exp Med. 2017:214:1111-28.

7. Köker MY, Camcioglu Y, van Leeuwen K, Kilic SS, Barlan I, Yilmaz M, et al. Clinical, functional, and genetic characterization of chronic granulomatous disease in 89 Turkish patients. J Allergy Clin Immunol. 2013;132(1156-63):e5.

8. Wolach B, Gavrieli R, de Boer M, van Leeuwen K, Berger-Achituv S, Stauber T, et al. Chronic granulomatous disease: clinical, functional, molecular, and genetic studies. The Israeli experience with 84 patients. Am J Hematol. 2017;92:28-36.

9. van den Berg JM, van Koppen E, Ahlin A, Belohradsky BH, Bernatowska E, Corbeel L, et al. Chronic granulomatous disease: the European experience. PLoS ONE. 2009;4:e5234.

10. Fattahi F, Badalzadeh M, Sedighipour L, Movahedi M, Fazlollahi MR, Mansouri SD, et al. Inheritance pattern and clinical aspects of 93 Iranian patients with chronic granulomatous disease. J Clin Immunol. 2011;31:792-801.

11. Kulkarni M, Hule G, de Boer M, van Leeuwen K, Kambli P, Aluri J, et al. Approach to molecular diagnosis of chronic granulomatous disease (CGD): an experience from a large cohort of 90 Indian patients. J Clin Immunol. 2018;38:898-916. 
12. Weel EA, Redekop WK, Weening RS. Increased risk of malignancy for patients with chronic granulomatous disease and its possible link to the pathogenesis of cancer. Eur J Cancer. 1996;32A:734-5.

13. Lugo Reyes SO, Suarez F, Herbigneaux RM, Pacquement H, Reguerre Y, Riviere JP, et al. Hodgkin lymphoma in 2 children with chronic granulomatous disease. J Allergy Clin Immunol. 2011;127(2):543-4.

14. Schappi MG, Jaquet V, Belli DC, Krause KH. Hyperinflammation in chronic granulomatous disease and anti-inflammatory role of the phagocyte NADPH oxidase. Semin Immunopathol. 2008:30:255-71.

15. Marciano BE, Rosenzweig SD, Kleiner DE, Anderson VL, Darnell DN, AnayaO'Brien S, et al. Gastrointestinal involvement in chronic granulomatous disease. Pediatrics. 2004;114:462-8.

16. Chou J, Hsu JT, Bainter W, Al-Attiyah R, Al-Herz W, Geha RS. A novel mutation in NCF2 associated with autoimmune disease and a solitary late-onset infection. Clin Immunol. 2015;161:128-30.

17. Maddah M, Fazlollahi MR, Shiari R, Shahram F, Mamishi S, Babaie D, et al. Lupus erythematosus and chronic granulomatous disease: report of four Iranian patients with AR-CGD and one XL-CGD. Iran J Allergy Asthma Immunol. 2019;18:452-8. https://doi.org/10.18502/ijaai.v18i4.1426.

18. De Ravin SS, Naumann N, Cowen EW, Friend J, Hilligoss D, Marquesen $\mathrm{M}$, et al. Chronic granulomatous disease as a risk factor for autoimmune disease. J Allergy Clin Immunol. 2008;122:1097-103.

19. Vowells SJ, Sekhsaria S, Malech HL, Shalit M, Fleisher TA. Flow cytometric analysis of the granulocyte respiratory burst: a comparison study of fluorescent probes. J Immunol Methods. 1995;178:89-97.

20. Petty RE, Southwood TR, Manners P, Baum J, Glass DN, Goldenberg J, et al. International league of associations for rheumatology classification of juvenile idiopathic arthritis: second revision, Edmonton, 2001. J Rheumatol. 2004;31:390-2.

21. Tronconi E, Miniaci A, Pession A. The autoimmune burden in juvenile idiopathic arthritis. Ital J Pediatr. 2017:43:56.

22. Vignesh P, Rawat A, Kumar A, Suri D, Gupta A, Lau YL, et al. Chronic granulomatous disease due to neutrophil cytosolic factor (NCF2) gene mutations in three unrelated families. J Clin Immunol. 2017;37:109-12.

23. Badalzadeh M, Fattahi F, Fazlollahi MR, Tajik S, Bemanian MH, Behmanesh $F$, et al. Molecular analysis of four cases of chronic granulomatous disease caused by defects in NCF-2: the gene encoding the p67-phox. Iran J Allergy Asthma Immunol. 2012;11:340-4.

24. Magnani A, Brosselin P, Beaute J, de Vergnes N, Mouy R, Debre M, et al. Inflammatory manifestations in a single-center cohort of patients with chronic granulomatous disease. J Allergy Clin Immunol. 2014;134(655-62):e8.

25. Liu TC, Stappenbeck TS. Genetics and pathogenesis of inflammatory bowel disease. Annu Rev Pathol. 2016;11:127-48.

26. Marks DJ, Miyagi K, Rahman FZ, Novelli M, Bloom SL, Segal AW. Inflammatory bowel disease in CGD reproduces the clinicopathological features of Crohn's disease. Am J Gastroenterol. 2009;104:117-24.

27. Muise AM, Xu W, Guo CH, Walters TD, Wolters VM, Fattouh R, et al. $\mathrm{NADPH}$ oxidase complex and IBD candidate gene studies: identification of a rare variant in NCF2 that results in reduced binding to RAC2. Gut. 2012;61:1028-35.

28. Dhillon SS, Fattouh R, Elkadri A, Xu W, Murchie R, Walters T, et al. Variants in nicotinamide adenine dinucleotide phosphate oxidase complex components determine susceptibility to very early onset inflammatory bowel disease. Gastroenterology. 2014;147(680-9):e2.

29. Jostins L, Ripke S, Weersma RK, Duerr RH, McGovern DP, Hui KY, et al. Host-microbe interactions have shaped the genetic architecture of inflammatory bowel disease. Nature. 2012;491:119-24.

30. AlKhater SA. CNS vasculitis and stroke as a complication of DOCK8 deficiency: a case report. BMC Neurol. 2016;16:54.

31. Schmidt RE, Grimbacher B, Witte T. Autoimmunity and primary immunodeficiency: two sides of the same coin? Nat Rev Rheumatol. 2017;14:7-18.

32. Sadrosadat T, Ziaee V, Aghighi Y, Moradinejad MH, Movahedi M. Presence of a juvenile idiopathic arthritis and chronic granulomatous disease in a child. Iran J Pediatr. 2015;25:e365.

33. Graham DSC, Morris DL, Bhangale TR, Criswell LA, Syvanen AC, Ronnblom $L$, et al. Association of NCF2, IKZF1, IRF8, IFIH1, and TYK2 with systemic lupus erythematosus. PLoS Genet. 2011;7:e1002341.

34. Kim-Howard X, Sun C, Molineros JE, Maiti AK, Chandru H, Adler A, et al. Allelic heterogeneity in NCF2 associated with systemic lupus erythematosus (SLE) susceptibility across four ethnic populations. Hum Mol Genet. 2014;23:1656-68.

35. Armstrong DL, Eisenstein M, Zidovetzki R, Jacob CO. Systemic lupus erythematosus-associated neutrophil cytosolic factor 2 mutation affects the structure of NADPH oxidase complex. J Biol Chem. 2015;290:12595-602.

36. Jacob CO, Eisenstein M, Dinauer MC, Ming W, Liu Q, John S, et al. Lupusassociated causal mutation in neutrophil cytosolic factor 2 (NCF2) brings unique insights to the structure and function of NADPH oxidase. Proc Natl Acad Sci U S A. 2012;109:E59-67.

37. Zhou Y, An LL, Chaerkady R, Mittereder N, Clarke L, Cohen TS, et al. Evidence for a direct link between PAD4-mediated citrullination and the oxidative burst in human neutrophils. Sci Rep. 2018;8:15228.

38. Romero V, Fert-Bober J, Nigrovic PA, Darrah E, Haque UJ, Lee DM, et al. Immune-mediated pore-forming pathways induce cellular hypercitrullination and generate citrullinated autoantigens in rheumatoid arthritis. Sci Transl Med. 2013;5:209ra150.

39. Eggleton P, Haigh R, Winyard PG. Consequence of neo-antigenicity of the 'altered self'. Rheumatology (Oxford). 2008;47:567-71.

40. Hultqvist M, Olsson LM, Gelderman KA, Holmdahl R. The protective role of ROS in autoimmune disease. Trends Immunol. 2009;30:201-8.

\section{Publisher's Note}

Springer Nature remains neutral with regard to jurisdictional claims in published maps and institutional affiliations.

\footnotetext{
Ready to submit your research? Choose BMC and benefit from:

- fast, convenient online submission

- thorough peer review by experienced researchers in your field

- rapid publication on acceptance

- support for research data, including large and complex data types

- gold Open Access which fosters wider collaboration and increased citations

- maximum visibility for your research: over 100M website views per year
}

At $\mathrm{BMC}$, research is always in progress.

Learn more biomedcentral.com/submissions 